\title{
Reporting of Medical Errors in Autopsied Cases
}

\author{
Bikash Sah, ${ }^{1}$ B. N. Yadav, ${ }^{1}$ Shivendra Jha, ${ }^{1}$ Ashok Ayer, ${ }^{2}$ Ajay Kumar Yadav ${ }^{3}$ \\ 'Department of Forensic Medicine \& Toxicology, ${ }^{2}$ Department of Conservative Dentistry \& Endodontics, \\ ${ }^{3}$ Department of General Practice \& Emergency medicine, B. P. Koirala Institute of Health Sciences, Sunsari, \\ Nepal.
}

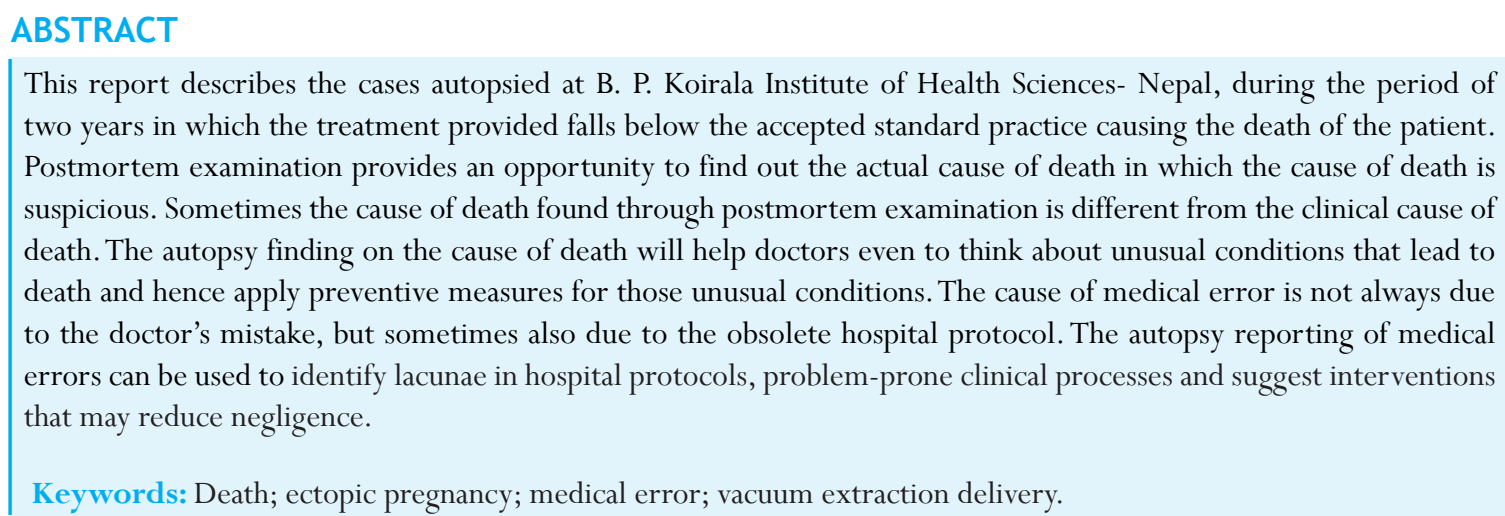

\section{INTRODUCTION}

Liability for negligence arises if the following conditions are satisfied. Duty: Existence of the doctor's duty of care to the plaintiff, based on the existence of the doctorpatient relationship.Dereliction: Failure on the part of the doctor to maintain the applicable standard of care and skill, i.e. a breach of the duty caused by the defendant's negligent act or omission. Direct Causation: Damages so occurred should be directly related to the dereliction, i.e. a direct link between the defendant's act or omission and an injury suffered by the plaintiff. Damage: It should be of a type that would have been foreseen by a reasonable physician, i.e. pain and suffering, disability and disfigurement, wrongful death etc. ${ }^{1}$ If the patient suffers harm, the health professional may be liable in tort law if the plaintiff can establish that the breach of the duty of care led to the reasonably foreseeable damage.Malpractice data can be used to identify problem-prone clinical processes and suggest interventions, identify potentially preventable sources of medical injury that may reduce negligence. ${ }^{2}$ The combination of ethical duties and legal responsibility form the foundation of professional and legal accountability. An editorial in 'Lancet' considered Medical errors worse than a crime. ${ }^{3}$

\section{CASE 1}

A 19-years-old pregnant lady from remote hill region was admitted in the gynaecology department of the hospital for delivery of her first baby at term. She was taken to the toilet by her husband for urination where she delivered the baby into the pan of the toilet and the infant died (Fig. 1). The husband mentioned that they were not instructed for urination at the bedside and filed the case of accidental death of the baby. Postmortem examination revealed $41 \mathrm{~cm}$ length male fetus with 29 $\mathrm{cm}$ head circumference. A contusion is present on the nose. Frontal bone is depressed into the cranial cavity compressing the underlying brain parenchyma. Lungs are in an expanded state in the thoracic cavity. On squeezing the lungs under water, some air bubbles come out on the water surface, suggesting the baby had respired after delivery. The cause of death was a head injury by blunt force.

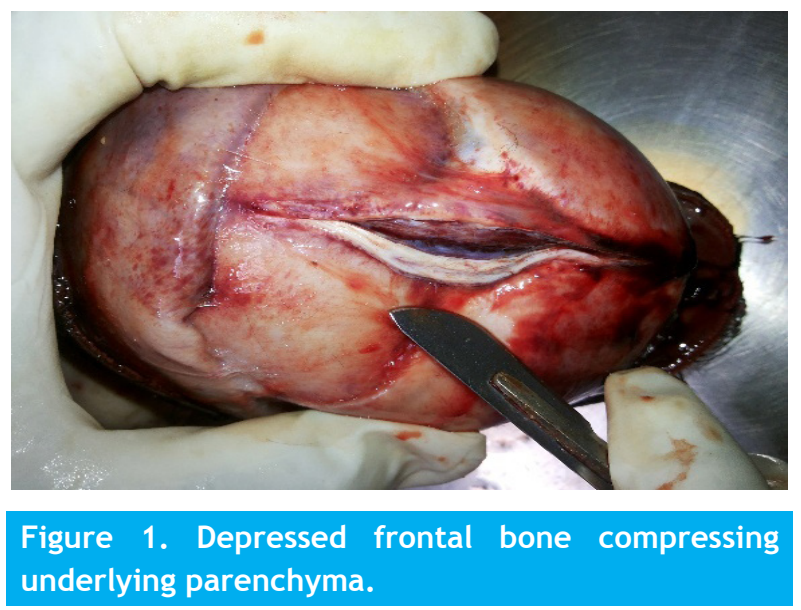

Correspondence: Bikash Sah, Department of Forensic Medicine \& Toxicology, B. P. Koirala Institute of Health Sciences, Dharan,Sunsari, Nepal. Email: bikashsa01@ gmail.com, Phone: +9779845224780 . 
CASE 2

A 26-years-old female had undergone post-abortion care at a private clinic after the medical abortion of one-month conception. She died after 22 days of postabortion care. She had experienced lower abdominal pain 24 hours prior to her death for which she took an oral analgesic. A post-mortem examination revealed faint post mortem staining on her back except at the areas of contact flattening. Blood was present at the vaginal opening. Approximately 4 litres of blood and blood clots were present in the pelvic cavity (Fig. 2). Internal organs were pale. Perforation was present on the left fallopian tube with a blackish mass at the torn site. The cause of death was a hemorrhagic shock as a result of rupture of the fallopian tube due to enlargement of ectopic pregnancy. The treating doctor was arrested from his clinic and was kept in police custody for investigation.

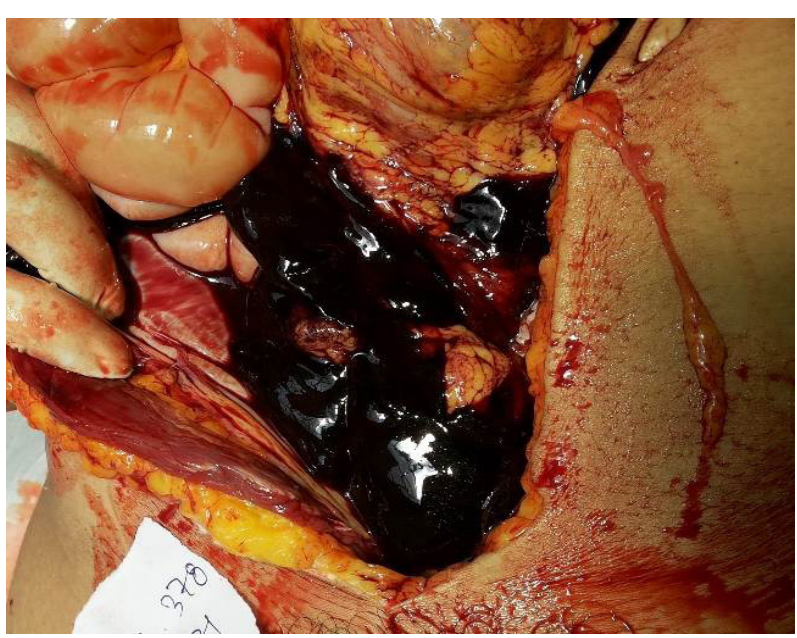

Figure2. Pelvic cavity with blood and blood clots.

CASE 3

An alleged history of the death of a 47 years old man given by his relatives was due to negligence of the doctor following treatment for a headache in a private clinic. He was conscious at 10:00 AM while visiting the clinic, but during the treatment, he went unconscious at 11:00 AM and did not return to conscious state and was discharged at 08:00 PM in unconscious state with the explanation that he was on sedative and would return to consciousness later on but was declared dead at 03:00 $\mathrm{AM}$ of the next day at another private clinic. His clinical check-up report of the $1^{\text {st }}$ clinic had shown his BloodPressure as $160 / 90 \mathrm{mmHg}$.

On post-mortem examination, subdural and subarachnoid hematoma were present in the frontoparieto-temporo-occipital region of the right cerebrum.
(Fig. 3). The internal wall of the stomach was congested with approximately $20 \mathrm{ml}$ of yellowish fluid. Liver and kidneys were congested. The spleen was enlarged. The cause of death was brain haemorrhage on right cerebral hemisphere as a result of hypertension.

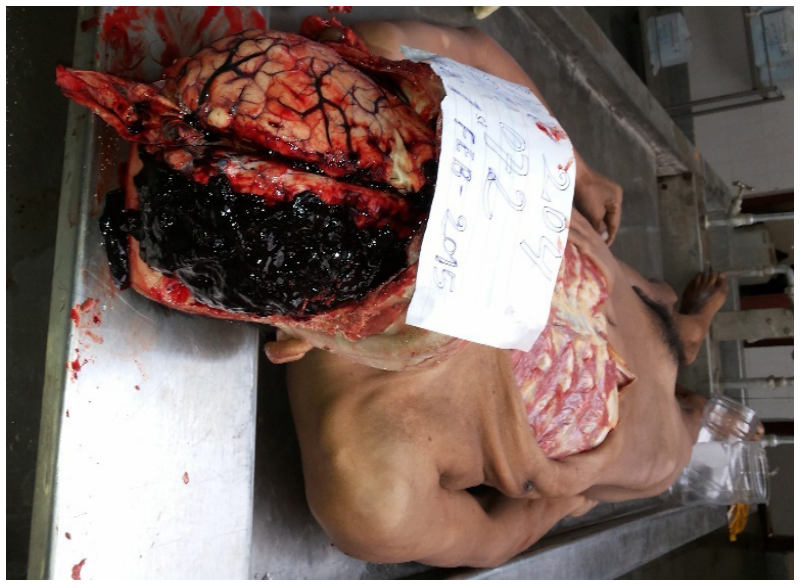

Figure 3. Subdural hematoma in front-parietotemporo-occipital region of right cerebrum.

CASE 4

Delivery of a male fetus from a normal full term pregnant lady was attempted by vacuum extraction followed by caesarian section. The child died during delivery and the relatives of the lady alleged that the child died due to the negligence of a doctor. Examination of the case record brought by police from the hospital revealed the following: Ultrasonography report showed a live fetus in cephalic presentation with normal cardiac activity and fetal movement and no gross congenital anomaly. Mother's vitals were stable at the time of admission. Admission records did not reveal informed consent for surgical intervention, except a blanket consent from her relative.

Postmortem examination had shown bluish discolorations on all nail beds and fingertips of both hands. Crownheel length was $55 \mathrm{~cm}$, head circumference was 34 $\mathrm{cm}$, chest circumference was $31 \mathrm{~cm}$, abdominal girth was $31 \mathrm{~cm}$. Blood stained fluid was present between the skull and scalp. Dark meconium was present in the large intestine. Stomach was empty. Right lung texture was similar to liver. Both lungs had a sharp border and were not inflated. Massive diffuse sub-scalp hematoma was present. Skull bones were in a partially deformed state (Fig. 4). Brain parenchyma was also deformed with a brain haemorrhage. The cause of death was shock as a result of injury to scalp, skull and brain produced during the process of birth more likely to be seen in the unskilled vacuum delivery. 


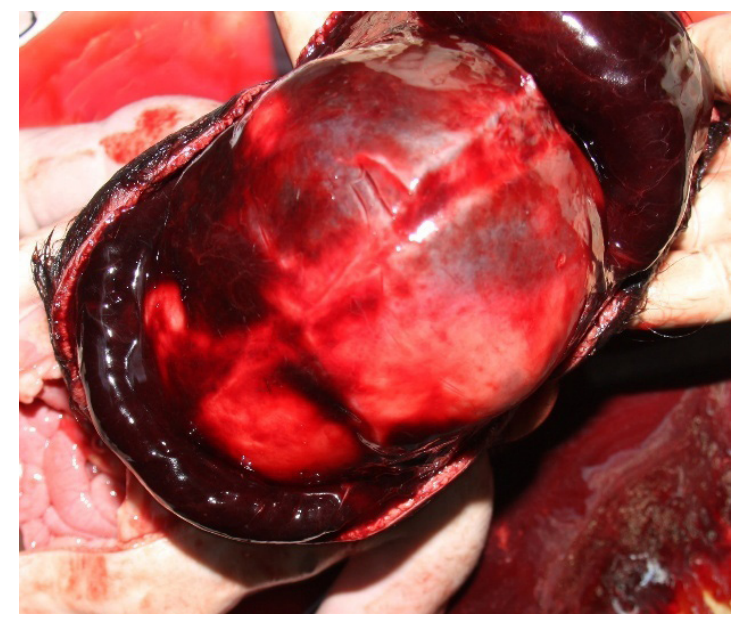

Figure 4. Fracture of right parietal bone with diffuse subscalp hematoma.

\section{DISCUSSION}

Medical errors that produce adverse effects form the basis of a sizeable proportion of claims for negligence. ${ }^{4}$ However, many of these are potentially preventable. An autopsy is an efficient method of clarifying medical malpractice claims..$^{5-7}$ Reporting of the autopsy in medical error and discussion with the physician, surgeons and medical establishment should be a routine process in any hospital setup. This is important to understand better for the cases without any plausible explanation. Moreover, medical record keeping and discussion of medical error/ negligence will also help in scientific evaluation and review of a patient management issue. Negligence is a preventable mistake. There must be a causal connection between a mistake and an injury, and this causal connection must be stated without reasonable doubt. ${ }^{8}$ An American study on evaluation of autopsy reports in litigation cases has shown that the doctors accused of medical malpractice do not have to fear the autopsy. ${ }^{9}$ Fear of autopsy findings is an obstacle to the pursuit of excellence through uninhibited outcome analysis. Despite knowing its importance, autopsy report discussion is rarely done among the concerned departments. It is yet to develop into the proper process in the hospital and clinics.

(Case-1): As per history given by the parents in the first case, they were counselled that this type of incidence is very rare and nothing can be done to prevent it. The parents were fully convinced that this was a case of an accident and the case was closed. If the case would have been further proceeded, some steps might have been taken from both doctor and hospital administration side to prevent such incidents in the future. There is nothing worse for any parent than the loss of a child and in coming to terms with such a loss, it's important for parents to seek answers and information as to why their child died during pregnancy or childbirth. The infant death could have been potentially avoided if the mother had been warned about the risk of precipitate labour during urination and if there had been timely and immediate access to advanced support. There are an increasing number of similar incidences; in Delhi (India) a pregnant mother went to hospital toilet unaccompanied and she delivered a baby which was stuck in the commode. ${ }^{10}$ An incidence took place in Uttar Pradesh (India) when a Nepalese woman gave birth to a baby which accidently passed through toilet of running train and fell on the train track, fortunately, baby survived due to prompt medical action. ${ }^{11}$ An event in the United Kingdom took place when a baby was born in toilet died at Royal Cornwall Hospital in Truro, and there were chances of survival if the mother had been on a labour suit. ${ }^{12}$ Changes in arrangement and safety protocols may prevent such incidences in future. Even though it is difficult to establish negligence on behalf of doctors and staff, but medical establishment and the entire team must take the incidence seriously and ensure the safety of both mother and newborn babies in the future.

(Case-2): More rarely an embryo may implant in the cervix, on an ovary, on the spleen or liver, in the cul-desac, on the abdominal wall or within the broad ligament. ${ }^{13}$ Although the fertilized egg is not cradled within the uterus, the embryo continues to grow and expand. Without treatment, the fallopian tube can rupture and can cause serious problems and sometimes death, as was in this case. In this case, the lady would have been saved if she would have visited the clinic on the very day, she experienced the pain. This would have been possible if the doctor would have considered an ectopic pregnancy in this case and would have warned the lady about its dangerous complication with especial emphasis for immediate follow up after any problem (like abdominal pain in this case). At the same time, the doctor would have been saved from his arrest if his prescription slip would have contained one simple statement in the local language that is "Immediately contact if any problem is seen/perceived."Clinicians should consider the diagnosis of ectopic pregnancy in any woman in the childbearing age with a history of secondary amenorrhea and who has abdominal or pelvic pain, vaginal bleeding, or both. ${ }^{14}$ Only $50 \%$ of patients present with the classic clinical triad of ectopic pregnancy i.e., pain, amenorrhea, and vaginal bleeding. ${ }^{15}$

(Case-3): Death, in this case, occurred due to the gross absence of knowledge and care in making a diagnosis and 
institution of proper treatment and physical care. The doctor didn't give proper instructions during discharge, and no referral was made. Negligent adverse effects was an injury caused by the failure to meet the standards of an average physician or institution. ${ }^{16}$ The doctor is the prime person who is responsible for history, physical examination, investigation, treatment plan, consent form, operative procedures, medication, discharge and referrals. It is the responsibility of the medical establishment and doctor to provide proper training to nursing and paramedical staff so that they will be aware of and be able to explain even rare complication for a particular condition to ensure that no negligence occurs.

(Case-4): Cause of death as mentioned by the doctor attending the delivery was due to the strangulation of the fetus by umbilical cord, but after autopsy, it was found to be due to shock as a result of injury to scalp, skull and brain produced during the process of birth more likely to be seen in the unskilled vacuum delivery. The doctor may have inappropriately attempted to perform a vacuum delivery, poorly handled baby and failed to recommend the caesarian section on time, thus, failing to meet the standard of care.The clinician must be trained and should know when to abandon the procedure and resort to caesarian section. A vacuum extraction tool, although beneficial during difficult deliveries can lead to birth injuries even death if misused. One of some absolute contraindications for Vacuum-Assisted Vaginal Delivery is a failure to obtain informed consent from the patient. ${ }^{17}$ Once the obstetric care provider has confirmed that the patient is an appropriate candidate for an operative vaginal delivery, informed consent should be obtained. Either way, the potential risks, benefits, and alternatives for operative vaginal delivery should be discussed, and the discussion should be clearly documented in the medical record. For the consent during assisted vaginal deliveries, emergency caesarian section and perineal repair prior to emergency procedures, there is scope to allow verbal consent to be obtained when it is considered to be in the interest of the woman or baby. However, if time allows written consent should be obtained for all such operations under general or regional anaesthesia. In the emergency situation, verbal consent should be obtained which should be witnessed by another care professional. Obstetricians and the witness to verbal consent must record the decision and the reasons for proceeding to any emergency delivery without written consent. ${ }^{18}$

The precise evaluation of autopsy reports in cases of medical malpractice is also a task of forensic medicine and toxicology as a contribution to increase patient safety by the identification and reporting of errors. ${ }^{19}$

\section{CONCLUSIONS}

Case- 1 could have been saved with simple instruction, case- 2 could have been saved if the doctor would have considered the chance of ectopic pregnancy in every case of abortion case- 3 has shown the gross absence of knowledge and care. Case- 4 has shown the importance of informed consent and maintaining medical record. The autopsy data are of special relevance for the identification, evaluation and prevention of errors and they are a valuable source of information for improvement of patient safety. At the same time, it is essential to increase awareness among patients to differentiate between an accident and a medical error and also to realize that an unborn child is legally part of the mother and, therefore, the mother is an actual victim of any negligence that causes any damage and/or death of the unborn.

\section{REFERENCES}

1. Reddy NKS, Murty OP: Essential of Forensic Medicine and Toxicology. $33^{\text {rd }}$ ed. Hyderabad: Jaypee Brothers Medical Publishers; 2015.

2. Kravitz RL, Rolph JE, McGuigan K. Malpractice claims data as a quality improvement tool. I. Epidemiology of error in four specialties. JAMA. 1991;266(15):2087-92. [PubMed]

3. Ferner RE, Aronson JK. Medication errors, worse than a crime.Lancet. 2000;355(9208):947-8.[Link]

4. The Lancet. Zeroing in on medication errors. Lancet. 1997;349(9049):369.[Link]

5. Perkins GD, McAuley DF, Davies S, Gao F. Discrepancies between clinical and postmortem diagnoses in critically ill patients: an observational study. Crit Care. 2003;7(6):R129-32.[PubMed]

6. Shojania KG, Burton EC. The vanishing nonforensic autopsy. N Engl J Med. 2008;358(9):873-5.[Link]

7. Shojania K, Burton E, McDonald K, Washington AE, Goldman L. The Autopsy as an Outcome and Performance Measure. Evidence Report/Technology Number 58. (prepared by the University of California at San FranciscoStanford, under contract no. 290-97-0013). Rockville, MD: Agency for Healthcare Research and Quality; 2002. [Full Text]

8. Hassan DA, Shehab AM, Kotb H. Alleged Medical 
Reporting of Medical Errors in Autopsied Case

Malpractice: A Retrospective Study of Forensic Evaluation of Cases in Cairo and Giza regions-Egypt (2009-2011). J Forensic Res. 2014;5(5):1.[Link]

9. Bove KE, Iery C; Autopsy Committee, College of American Pathologists. The role of the autopsy in medical malpractice cases, I: a review of 99 appeals court decisions. Arch patholLab Med. 2002;126(9):1023-31.[PubMed]

10. Tiwari S. 2 women give birth in hospital toilet. The Indian Express. 2015 Dec 31.[Link]

11. HT Correspondent. Miracle? Baby delivered inside railway toilet falls on track, survives. Hindustan times. 2015 Dec 30. [Link]

12. BBC News. Baby died after being born in hospital toilet, inquest hears. BBC News. 2015 Oct 7. [Link]

13. Cunningham FG. Williams Obstetrics. 20th ed. Connecticut, USA: Appleton \& Lange; 1997

14. van Mello NM, Mol F, Adriaanse AH, Boss EA, Dijkman $\mathrm{AB}$, Doornbos JP, et al. The METEX study: methotrexate versus expectant management in women with ectopic pregnancy: a randomised controlled trial. BMC Women's Health. 2008;8(1):10.[PubMed]

15. Shalev E, Peleg D, Tsabari A, Romano S, Bustan M.
Spontaneous resolution of ectopic tubal pregnancy: natural history. Fertil steril. 1995;63(1):15-9.[PubMed]

16. Hiatt HH, Barnes BA, Brennan TA, Laird NM, Lawthers AG, Leape LL, et al. A study of medical injury and medical malpractice. N Engl J Med. 1989;321(7):480-4.[Link]

17. Ali UA, Norwitz ER. Vacuum-assisted vaginal delivery. Rev Obstet Gynecol. 2009;2(1):5.[PMC free article]

18. Morris EP. Obtaining valid consent. Clinical governance advice no. 6). Royal College of Obstetricians \& Gynaecologists. 2015 Jan. [Link]

19. World Health Organization. World alliance for patient safety: WHO draft guidelines for adverse event reporting and learning systems: from information to action. Geneva: World Health Organization; 2005. [Full Article] 